\title{
Revision of Failed Open Reduction of Developmental Dysplasia of the Hip
}

\author{
Mohamed Mansour Elzohairy, MD, Mohamed Mahmoud Elhefnawy, MD, Hosam Mohamed Khairy, MD \\ Faculty of Human Medicine, Zagazig University, Zagazig, Egypt
}

\begin{abstract}
Background: The most common causes of re-dislocation after open reduction are inadequate exposure and failure to release the obstructing soft tissues inside and around the hip.

Methods: This clinical study included 33 consecutive children (34 hips) who underwent a revision surgery after failed open reduction of developmental dysplasia of the hip (DDH).

Results: According to the McKay clinical criteria, the results were good in 28 cases (82.4\%), fair in 4 cases (11.8\%), and poor in 2 cases with re-dislocation (5.9\%). Radiological results according to the modified Severin criteria were as follows: 28 hips (82.4\%) were identified as category 2 (good), 4 hips (11.8\%) category 4 (fair), and 2 hips (5.9\%) category 5 (poor).

Conclusions: Revision surgery for DDH is demanding and the long-term consequences are usually serious, but stable, concentric reduction should be obtained either at the first or second open reduction by addressing the causes of failure. Failed acetabulum remodeling and technical errors with inadequate soft-tissue release were the most common causes of failure in the primary operation. Based on the results, the outcome of revision surgery after failed open reduction for DDH was good.

Keywords: Revision, Failed, Open reduction, Developmental dysplasia, The hip
\end{abstract}

For developmental dysplasia of the hip (DDH), the aim of treatment is to obtain and maintain a stable, concentric reduction as early as possible. ${ }^{1,2)}$ However, uncommonly, open reduction fails and re-dislocation occurs. ${ }^{3,4)}$ The reported rate of failure or re-dislocation after open reduction through an anterolateral approach varies from $0 \%$ to $8 \%{ }^{5-7)}$ Re-dislocation after primary treatment of $\mathrm{DDH}$ is a serious complication and revision surgery after failed open reduction for DDH is technically demanding. ${ }^{802}$ Earlier studies have reported and highlighted the complications associated with the management of this difficult problem. ${ }^{1-4)}$

The hypothesis of the current study was that im-

Received October 28, 2019; Accepted May 6, 2020

Correspondence to: Mohamed Mansour Elzohairy, MD

Faculty of Medicine, Zagazig University Hospitals, Dr, Mansour Elzohairy Villa, 4 Zeid Ben Sabet Street, University Villas, Zagazig, 44511, Al Sharqia, Egypt

Tel: +20-112-446-7856, +20-552-374-060

E-mail: elzohairy.me@gmail.com proper surgical techniques and lack of experience would be the causes of re-dislocation and failure of open reduction for DDH. In the current study, we sought to answer the following query: are the revision procedure and reduction worthy operations for later development of normal hip joint? Also we focused on investigating the possible causes of re-dislocation and risk factors of failure, which should be addressed to avoid re-dislocation.

\section{METHODS}

We present in this study 33 patients (34 hips) who developed re-dislocation after an open reduction for $\mathrm{DDH}$ and underwent second revision surgery for open reduction and relocation of the dislocated hip. Among the 33 patients, 28 were female and 5 were male patients ( 34 hips [21 left and 13 right] including 1 bilateral case), who were treated between December 2016 and January 2000. All cases had been treated initially at other hospitals except 4 cases that had been treated at our hospital. We included all cases that had re-dislocation or persistent dislocation after 
open reduction and had typical idiopathic DDH; excluded cases (15 cases) had neuromuscular or any other congenital disorders.

$\mathrm{McKay}^{21)}$ clinical criteria score was used for clinical evaluation. Anteroposterior and lateral radiographs of the pelvis, hip joint, and femur were obtained in all cases. The size and shape of the femoral head, the femoral neck length, and the degree of osteopenia of the proximal femur were evaluated. A modified Severin criteria was used for radiological evaluation. ${ }^{22,23)}$ We checked the evidence of avascular necrosis (AVN) of the femoral head. If AVN was noted, it was categorized before revision surgery for follow-up documentation. The Kalamchi classification ${ }^{24)}$ was used for radiological evaluation of AVN of the femoral head. Computed tomography and magnetic resonance imaging were performed in all cases.

\section{First Operation}

The patient's age at the time of the first operation ranged from 1.5 to 2.5 years (mean, 1.7 years).

\section{The Operative Details}

A bikini incision was used in 28 hips while the longitudinal Smith Peterson approach was used in 6 hips.

\section{Femoral Osteotomy}

A femoral osteotomy was performed through a lateral approach in 8 hips.

\section{Pelvic Osteotomy}

No pelvic osteotomy procedures were done in any cases. In 2 hips where 2 revisions were done, the first revision was an adductor tenotomy with closed reduction and the second was open reduction with a femoral osteotomy.

\section{Second Operation}

The patient's age at the time of revision surgery ranged from 2 to 4.5 years (mean, 3 years). The mean time interval between the first and second operation ranged from 0.5 years (6 months) up to 2.5 years (mean, 1.1 years). We followed a protocol, which recommended at least 6 months of interval between the first and second operations to achieve the greatest range of motion, to allow for maturation of the scar tissue (to become more stretchable), and to avoid extensive bleeding from the immature highly vascular tissue.

In 2 cases, re-revision was done at 2 months $(0.2$ years) after primary operation because of early dislocation in the spica associated with bad nursing. In the 2 cases, redislocation occurred after the second operation because of poor dissection due to extensive intraoperative bleeding from the immature fibrous tissue. A vascular surgeon was present during these operations, but no vascular injury occurred in both cases. The presence of a vascular surgeon was a precautionary measure due to the proximity of the medial dissection to the neurovascular trunk (femoral artery).

\section{The Operative Details}

A bikini incision was used in all cases. In 32 cases, we started with an adductor tenotomy for easier reduction of the head.

\section{Femoral Osteotomy through Lateral Approach}

Femoral osteotomy was primarily done in 5 cases (varus + derotation) and revised in 14 cases (in 10 cases due to excessive retroversion and in 4 cases due to undercorrection). Femoral shortening was done in 22 hips to decrease tension over the femoral head, which may lead to AVN. In 1 hip, femoral osteotomy was done at the first time and was not revised.

\section{Pelvic Osteotomy}

Salter's osteotomy was done in 11 cases for anterolateral coverage. Dega osteotomy was done in 10 hips for anteroposterior coverage of the head. Khairy Modified Lance Acetabuloplasty (KMLA) ${ }^{15)}$ was performed for correction of the deformed bipartite acetabulum in 13 hips, as we could not rely on the remodeling of the acetabulum in the revision procedure because of poor capsulorrhaphy, deformed labrum, which affects the femoral head stability inside the hip joint, and the injured acetabulum with a low growth potential.

\section{Intraoperative Findings}

The intraoperative findings include thick ligamentum teres (not cut in 7 cases), tight inferior capsule and labrum (not released in 14 hips), slanting acetabulum in 21 hips, bipartite acetabulum in 13 hips, deformed femoral heads in 3 hips (incongruous congruity), and tight hip in need of shortening of the femur in 22 hips.

\section{Postoperative Casting}

In all 33 patients, a spica cast was applied for 2.5 months, which was followed by the use of a broomstick plaster for 2 weeks and then bracing for 3 months except in 5 cases where the brace was worn for another 3 months.

\section{Statistical Analysis}

Statistical analysis was performed by using IBM SPSS 
Elzohairy et al. Revision of Failed Open Reduction of Developmental Dysplasia of the Hip

Clinics in Orthopedic Surgery • Vol. 12, No. 4, $2020 \bullet$ www.ecios.org

Table 1. Clinical and Radiological Outcomes

\begin{tabular}{lc}
\hline \multicolumn{1}{|c}{ Evaluation } & No. $(\%)$ \\
\hline McKay criteria & \\
\hline Good & $28(82.4)$ \\
\hline Fair & $4(11.8)$ \\
\hline Poor & $2(5.9)$ \\
\hline Kalamchi classification & \\
\hline 0 & $29(85.3)$ \\
\hline 1 & $1(2.9)$ \\
\hline 2 & $1(2.9)$ \\
\hline 3 & $1(2.9)$ \\
\hline 4 & $2(5.9)$ \\
\hline Modified Severin criteria & \\
\hline 1: Good & $28(82.4)$ \\
\hline 2: Fair & $4(11.8)$ \\
\hline 3: Poor & $2(5.9)$ \\
\hline Complication & $1(2.9)$ \\
\hline Sciatic nerve injury & $2(5.9)$ \\
\hline
\end{tabular}

ver. 20 (IBM Corp., Armonk, NY, USA). Qualitative data were presented as number and percentage. Quantitative data were presented as mean \pm standard deviation for parametric data or median and range for nonparametric data. The student $t$-test was used to compare the 2 groups. Spearman's correlation coefficients were used to test the correlation between variables. A $p<0.05$ was considered statistically significant.

\section{RESULTS}

At the last follow-up, both clinical evaluation and radiological assessment were done for all patients. The followup period varied from 5 years up to 7 years (mean, 5.9 years). According to the McKay clinical criteria, the clinical results were good in 28 cases (82.4\%), fair in 4 cases $(11.8 \%)$, and poor in 2 cases with recurrent dislocation (5.9\%). The radiographic results classified according to the modified Severin criteria were as follows: 28 hips (82.4 \%) in category 2 (good), 4 hips (11.8\%) in category 4 (fair), and 2 hips (5.9\%) in category 5 (poor).

The complications were as follows: sciatic nerve injury in 1 hip (2.9\%), re-dislocation in 2 hips (5.9\%), and complete AVN in 2 hips (5.9\%) (Table 1, Fig.1). There was no significant difference in age at the primary operation, but the age at the second operation, interval between operations, Severin score, and Kalamchi score were significantly lower in patients with good clinical scores.

Severin and Kalamchi scores were significantly correlated with each other and both scores were significantly
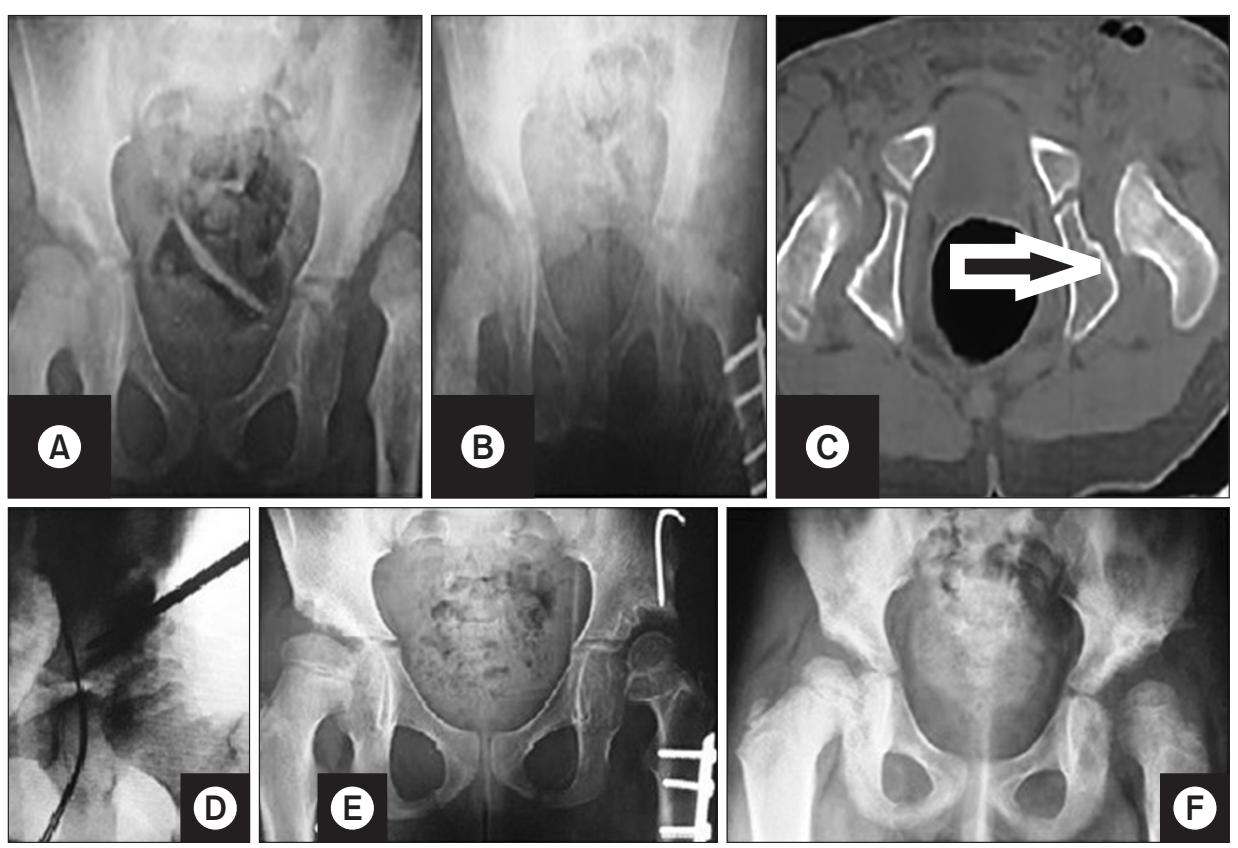

Fig. 1. Case presentation. (A) Developmental dysplasia of the left hip in a patient aged 2 years. (B) Immediate postoperative radiograph after open reduction and femoral derotation osteotomy. (C) Postoperative computed tomography scan of hip subluxation, showing insufficient reduction with bipartite acetabulum (arrow), which was the cause of re-dislocation. (D) Revision open reduction, Khairy modified lance acetabuloplasty osteotomy, and graft in site. (E) The graft was fixed by a Kirschner wire. (F) Five-year follow-up radiograph. 
positively correlated with age at the second operation and interval between operations. In other words, the longer the interval between the first and second operations, the higher the age at the second operation and the worse the prognosis (Tables 2 and 3).

\section{DISCUSSION}

There are many causes that lead to re-dislocation of the hip joint in patients with DDH after open reduction. Johari and Wadia ${ }^{6}$ divided the possible causes of failure of primary open reduction into 3 categories according to the timing of failure (immediate, delayed, and late failures).

\section{Immediate Failure}

hip dislocation is noticed in the immediate postoperative X-ray, which may be due to an approach-related error or inadequate exposure as in the medial approach in older children. ${ }^{6,24)}$ Technical errors of open reduction are associated with inability to release all obstructing soft tissues. ${ }^{4,6,9-12,24)}$ Technical errors of the procedures that should be performed in addition to open reduction include failure to perform an adequate femoral shortening osteotomy when needed especially with an acetabuloplasty that increases markedly the pressure on the femoral head, failure to correct a severely anteverted femoral head by a proper derotation osteotomy, and failure to correct coxa valga by a varus osteotomy. Correct displacement of the pelvic osteotomy is a more challenging task when performed in conjunction with the open reduction than as an isolated procedure, as control of the acetabulum is more difficult once the hip joint capsule has been opened. ${ }^{10-12)}$

\section{Delayed Failure}

Hip dislocation discovered after removal of a hip spica (at 4 to 6 weeks). Its causes could be inadequate capsulorrhaphy, which is the most common cause of delayed failure, inadequate stabilization of the pelvic osteotomy graft, and unstable fixation of the femoral osteotomy. ${ }^{11,13,18-20)}$ Inadequate immobilization due to early removal of a hip spica.

\section{Late Failure}

Dislocated or subluxated hip after a normal intervening period of relocation postoperatively, usually due to abnormal remodeling of the acetabulum or the proximal femur. In most cases, failure to get stable, concentric reduction at the first surgery is the result of errors in judgment or surgical techniques. ${ }^{2)}$ Most patients did not have a sufficient soft-tissue release or an adequate clearance of the structures, which prevented reduction of the head into

Table 2. Comparison between Good Outcome and Fair and Bad Outcomes

\begin{tabular}{lcccc}
\multicolumn{1}{c}{ Variable } & Good & Fair \& bad & Student $t$-test & $p$-value \\
Age at 1st operation & $1.67 \pm 0.52$ & $1.81 \pm 0.60$ & 1.017 & 0.215 \\
Age at 2nd operation & $2.85 \pm 0.95$ & $4.03 \pm 1.12$ & 2.387 & $0.025^{*}$ \\
Interval between operations & $0.91 \pm 0.28$ & $1.54 \pm 0.37$ & 3.034 & $0.002^{*}$ \\
\hline
\end{tabular}

Values are presented as mean \pm standard deviation.

*There was no significant difference with regard to age at the first operation, but patients with good outcome were significantly younger at the second operation with a shorter interval between operations. This indicates that the higher the age and the longer the interval between the first and the second revision operations, the worse the prognosis of revision for developmental dysplasia of the hip.

Table 3. Correlation of Severin and Kalamchi Scores

\begin{tabular}{lcccc}
\multicolumn{1}{c}{ Variable } & \multicolumn{2}{c}{ Severin } & & Kalamchi \\
\cline { 2 - 5 } & $r$ & $p$-value & $r$ & $p$-value \\
Age at 1st operation & 0.021 & 0.845 & 0.020 & 0.845 \\
Age at 2nd operation & 0.459 & $<0.001^{*}$ & 0.587 & 0.599 \\
Interval between operations & 0.412 & $<0.001^{*}$ & $<0.001^{*}$ \\
Kalamchi & 0.785 & $<0.001^{*}$ & \\
\hline
\end{tabular}

*Severin and Kalamchi scores were significantly correlated with each other and significantly positively correlated with age at the second operation and interval between operations. So this indicates that the higher the age and the interval between the first and the second revision operations, the worse the prognosis of revision for developmental dysplasia of the hip. 
the acetabulum. Our findings agree with those in other studies regarding the main cause of failure of reduction in primary surgery. ${ }^{1-4,14-20)}$ The success of capsulorrhaphy is essential in the first few weeks after open reduction; undue tension applied either posteriorly or anteriorly may lead to dehiscence or stretching. Failure to obtain proper tightness of the posterior capsule may result in posterior subluxation, especially if a Salter osteotomy is done simultaneously. Our findings in these aspects agree with those of Bos and Slooff ${ }^{3)}$ and McCluskey et al. ${ }^{2)}$ We also agree with earlier studies that intra-articular adhesions should be completely excised to allow reduction. ${ }^{16)}$ In the current study, the causes of failure of the primary open reduction were as follows: tight adductors in 32 cases, tight ligamentum teres (not cut in 7 cases), and the inferior capsule and labrum tight and not released in 14 hips. In cases with femoral osteotomy, excessive retroversion was the cause in 10 cases, undercorrection in 4 cases, deformed femoral head in 3 cases (incongruous congruity), and tight hip in need of shortening of the femur in 22 hips. There was slanting acetabulum in 21 hips and bipartite acetabulum in 13 hips. So we agree with other authors that failure of primary reduction could be attributed to technical errors with lack of experience in these difficult cases. As bipartite acetabulum can be expected radiologically and confirmed intraoperatively, the problem with bipartite acetabulum is different from acetabular dysplasia. In dysplasia, an increased acetabular index is observed without intra-articular deformities, whereas in bipartite acetabulum, there is an intra-articular deformity, which needs an osteotomy that addresses the intra-articular part of the deformity. Reconstructive pelvic osteotomies are either re-directional or reshaping osteotomies; the first concern lies with changing the orientation of the acetabulum and the second concern with changing the shape of the acetabulum. Most reshaping osteotomies depend on the triradiate cartilage as a fulcrum on which the acetabular roof is turned down as in Pemberton pelvic osteotomy. Lance acetabuloplasty starts just above the labrum acetabuli and extends above the subchondral bone inwards for $2-3 \mathrm{~cm}$, and then the roof is turned down.

Rejholec $^{18,19)}$ used combined pelvic osteotomy (Salter + Lance acetabuloplasty) in the treatment of bipartite acetabulum for correction of both orientation and shape of the acetabulum (in residual dysplasia) with the primary open reduction. In the study, Lance acetabuloplasty was used for correction of the shape of the normally oriented acetabulum but deformed by the subluxated femoral head in combined with revision open reduction. In the study, the final evaluation was dependent on the McKay clinical criteria and plain radiography.

We used the KMLA ${ }^{16)}$ in which we made a cut above the acetabulum that ends opposite to the margin between both parts of the bipartite acetabulum. We used KMLA, not Salter osteotomy, because the adhesions made the medial dissection on the inner pelvic table difficult, but in KMLA osteotomy, we dealt with the outer cortex. We did not encounter any incidence of lateral hip growth arrest, which could result in dysplasia of the acetabulum in any cases that underwent KMLA osteotomy, but we still need to investigate the long-term results in our next study to evaluate these cases.

Although we had promising, good results with the KMLA osteotomy technique for correction of the bipartite acetabulum and reforming of new bone coverage over the femoral head, we still need more time to confirm the last results and are waiting for more time for evaluation. The femoral osteotomy was done in 12 hips ( 5 new and 7 revisions) with shortening plus medial wedges to make varus alignment. Regarding the best time for the secondary surgery, we agree with other authors, ${ }^{2,24)}$ who recommended delaying the revision surgery until the soft tissues and skin have softened up and all hip motions have returned to prereduction ranges. It takes at least 6 months $(0.5$ year) for the scar tissue to become mature to prevent profuse bleeding from the immature scar tissue during surgery.

We followed these recommendations in our patients except in 2 cases, in which we discovered dislocation due to bad nursing during the postoperative casting period and thus reopened 2 months ( 0.2 year) after the primary surgery. In 1 case, reduction was successful but results were poor while in the other case, re-dislocation occurred because of poor dissection due to profuse intraoperative bleeding from the immature scar with failure and we had attendance of a vascular surgeon during the operation in both cases due to proximity to the neurovascular bundle.

Attendance of a vascular surgeon is not a mandatory protocol during revision $\mathrm{DDH}$ surgery, but it was a precautionary measure taken for the 2 cases with early revision, considering the risk of extensive intraoperative bleeding from the immature fibrous tissue. The surgery was performed with attendance of a vascular surgeon, but no vascular injury occurred in both cases.

There was no significant difference in age at first operation but the good clinical score group was significantly younger at the second operation and the interval between operations shorter, and the Severin score and Kalamchi score were lower. Severin and Kalamchi scores were significantly correlated with each other and both were significantly positively correlated with age at the second op- 
eration and interval between operations. In other words, the longer the interval between the first and the second operations and the higher the age at the second operation, the worse the prognosis was. These results were in accord with the results of Abouelnas et al., ${ }^{20)}$ who reported a positive correlation between the age of the patients and the 3 scores, but it was significant only with Kalamchi score.

In revision operations, the surgeon faces many challenges that are greater than those faced at first operations for DDH. The second line of surgical options or a backup plan should always be ready before surgery. Revision surgery for DDH can usually improve function, but rarely results in a healthy, normal joint, ${ }^{13,14)}$ and this has to be clearly clarified to the family preoperatively to avoid having angry parents postoperatively. ${ }^{2,3,13,24)}$ The main limitation of the present study is the absence of long-term follow-up, especially in the adult period with KMLA because there would be risks for iatrogenic physeal arrest on secondary ossification center around the labrum and AVN with too small osteotomy fragments with this technique. Therefore, we defer definitive conclusions until meaningful results based on long-term follow-up and collection of more cases are obtained. However, compared to the other long-term reports in the literature, we could expect less favorable long-term outcome of the revision surgery to the primary surgery. Although we could not expect excellent outcome in all revision cases, relocation of the dislocated femoral head is still mandatory to facilitate the growth of both femoral head and acetabulum.

Failure to obtain a stable, concentric reduction after primary open reduction of DDH is mainly due to the failed acetabulum remodeling, which is followed by technical errors with inadequate soft-tissue release. Based on the results of the current study, the outcome of revision surgery after failed open reduction for DDH can be expected to be good. KMLA is a good solution for correction of a deformed bipartite acetabulum as a residual of hip dysplasia. The procedure is simple but needs to be precisely designed for the defective area. It could be done combined with open reduction with or without a femoral osteotomy. However, the efficacy this technique still requires longer time to be fully evaluated.

\section{CONFLICT OF INTEREST}

No potential conflict of interest relevant to this article was reported.

\section{ACKNOWLEDGEMENTS}

We would like to thank Dr.Wael Galal Abd Elazem, who is a lecturer at community medicine Zagazig University, Egypt, for his sincere effort and help in editing this manuscript.

\section{REFERENCES}

1. Hsieh SM, Huang SC. Treatment of developmental dysplasia of the hip after failed open reduction. J Formos Med Assoc. 1998;97(11):763-9.

2. McCluskey WP, Bassett GS, Mora-Garcia G, MacEwen GD. Treatment of failed open reduction for congenital dislocation of the hip. J Pediatr Orthop. 1989;9(6):633-9.

3. Bos CF, Slooff TJ. Treatment of failed open reduction for congenital dislocation of the hip: a 10-year follow-up of 14 patients. Acta Orthop Scand. 1984;55(5):531-5.

4. Kershaw CJ, Ware HE, Pattinson R, Fixsen JA. Revision of failed open reduction of congenital dislocation of the hip. J Bone Joint Surg Br. 1993;75(5):744-9.

5. Kampa RJ, Prasthofer A, Lawrence-Watt DJ, Pattison RM. The internervous safe zone for incision of the capsule of the hip: a cadaver study. J Bone Joint Surg Br. 2007;89(7):971-6.

6. Johari AN, Wadia FD. Revision surgery for developmental dysplasia of the hip. Indian J Orthop. 2003;37(4):233-6.

7. Mayo KA, Trumble SJ, Mast JW. Results of periacetabular osteotomy in patients with previous surgery for hip dysplasia. Clin Orthop Relat Res. 1999;(363):73-80.

8. Ponseti I. Causes of failure in the treatment of congenital dislocation of the hip. J Bone Joint Surg. 1944;26(4):775-92.

9. Chidambaram S, Abd Halim AR, Yeap JK, Ibrahim S. Revision surgery for developmental dysplasia of the hip. Med J Malaysia. 2005;60 Suppl C:91-8.

10. Chmielewski J, Albinana J. Failures of open reduction in developmental dislocation of the hip. J Pediatr Orthop B. 2002;11(4):284-9.

11. Haidar RK, Jones RS, Vergroesen DA, Evans GA. Simultaneous open reduction and Salter innominate osteotomy for developmental dysplasia of the hip. J Bone Joint Surg Br. 1996;78(3):471-6.

12. Barrett WP, Staheli LT, Chew DE. The effectiveness of the Salter innominate osteotomy in the treatment of congenital dislocation of the hip. J Bone Joint Surg Am. 1986;68(1):7987. 
Elzohairy et al. Revision of Failed Open Reduction of Developmental Dysplasia of the Hip

Clinics in Orthopedic Surgery • Vol. 12, No. 4, $2020 \bullet$ www.ecios.org

13. Dimitriou JK, Cavadias AX. One-stage surgical procedure for congenital dislocation of the hip in older children: longterm results. Clin Orthop Relat Res. 1989;(246):30-8.

14. Sankar WN, Young CR, Lin AG, Crow SA, Baldwin KD, Moseley CF. Risk factors for failure after open reduction for DDH: a matched cohort analysis. J Pediatr Orthop. 2011;31(3):232-9.

15. Herold HZ. Pediatric update \#9: revision surgery in congenital dislocation of the hip. Orthop Rev. 1989;18(8):90310.

16. Khairy HM. Modified lance acetabuloplasty for deformed bipartite acetabulum in residual hip dysplasia. Egypt Orthop J. 2014;24(4):1-12.

17. Weber M, Wirtz D, Jaeschke C, Niethard FU. Growth disorders of the acetabular roof after acetabuloplasty in congenital hip dysplasia. Z Orthop Ihre Grenzgeb. 1998;136(6):52533.

18. Rejholec M. Combined pelvic osteotomy for deformed dysplastic acetabula: a 5-year prospective study. J Orthop Surg (Hong Kong). 2007;15(3):347-51.

19. Rejholec M. Combined pelvic osteotomy for the bipartite acetabulum in late developmental dysplasia of the hip: a tenyear prospective study. J Bone Joint Surg Br. 2011;93(2):25761.

20. Abouelnas BA, Zaghloul K, Saied AM. Revision surgery for developmental dysplasia of the hip (DDH), midterm result. Egypt Orthop J. 2018;53 (supple 1):45-50.

21. McKay DW. A comparison of the innominate and the pericapsular osteotomy in the treatment of congenital dislocation of the hip. Clin Orthop Relat Res. 1974;(98):124-32.

22. Severin EA. Contribution to the knowledge of congenital dislocation of the hip joint: late results of closed reduction and arthrographic studies of recent cases. Stockholm: Norstedt; 1941. 1-142.

23. Ward WT, Vogt M, Grudziak JS, Tumer Y, Cook PC, Fitch RD. Severin classification system for evaluation of the results of operative treatment of congenital dislocation of the hip: a study of intraobserver and interobserver reliability. J Bone Joint Surg Am. 1997;79(5):656-63.

24. Kalamchi A, MacEwen GD. Avascular necrosis following treatment of congenital dislocation of the hip. J Bone Joint Surg Am. 1980;62(6):876-88. 